International Journal of Engineering \&Technology, 8(4) (2019) 636-641
International Journal of Engineering \& Technology
SPC
Website: www.sciencepubco.com/index.php/IJET
Research paper

\title{
The design of a microcontroller-based automatic liquid level control system
}

\author{
Saleh Ahmad ${ }^{1}$ *, Abd Al Rahman Abu Ebayyeh ${ }^{2}$, Abdulaziz Alhashmi ${ }^{1}$ \\ ${ }^{1}$ Abu Dhabi Polytechnic, UAE \\ ${ }^{2}$ Brunel University, $U K$ \\ *Corresponding author E-mail: saleh.ahmad@adpoly.ac.ae
}

\begin{abstract}
In this paper, a liquid level control system was designed and fabricated. Control of the liquid level was accomplished by adjusting the supply voltage to a centrifugal pump that is delivering the liquid from a reservoir tank to an upper tank. The liquid level control system was implemented on an ATmega328 microcontroller. Three control strategies are implemented, manual mode, ON/OFF controller, and PID controller. Serial communication is used to establish the communication between the ATmega328 and a computer running MATLAB software. A graphical user interface was constructed to allow users to interact with the system. The system model is obtained using an experimental approach. The obtained model is then used for the design of the PID controller. Experimental results are provided to highlight the performance of the implemented controllers.
\end{abstract}

Keywords: Liquid Level Control; ON/OFF Controller; PID Controller; MATLAB GUI.

\section{Introduction}

Liquid level control is a very common application in the process industry such as in food processing, pharmaceutical industry, water purification, chemical industry, and many more. In this paper, a liquid level control system that is composed of two tanks, an overhead tank and a reservoir, is designed and implemented. The system is controlled with a custom designed graphical user interface (GUI) that allows for two control methods; namely, ON/OFF Controller and PID Controller. The designed system is used as a laboratory equipment for training students in the process control area.

Automatic control systems have been developing in the course of the last 150 years [1]. Liquid level control systems began as basic mechanical feedback devices and have advanced into complex electronic and computer-controlled systems [2]-[6]. This paper presents the development of an apparatus that demonstrates the concepts of automatic control of liquid level in a tank that is being randomly drained. The random draining of the upper tank simulates the unpredictable disturbances that are inevitable in real-life applications. In this work, the automatic liquid level control is accomplished by sensing the liquid level and then manipulating the supply voltage to the pump with two points ON/OFF controller or PID controller.

The education of students in the control systems area is one of the main objectives of Abu Dhabi Polytechnic, UAE. The developed automatic liquid level control system will be used as a teaching aid so that the vocational students at Abu Dhabi polytechnic can interact with a dynamic control system operating in real-time.

The design and analysis of the controller is typically based on the plant mathematical model, which could be very difficult to formulate analytically. The mathematical model of the system is derived from experimental data with the help of MATLAB software. The obtained system model is then used for designing the Proportional Integral Derivative (PID) controller. PID controllers are simple and reliable; therefore, they are commonly used in industrial feedback control systems. The performance analysis of PID controllers have been discussed in [8]-[11]. It is also the most adopted controllers in the industry due to their low cost of implementation and maintenance [7].

This paper is organized as follows: Section 2 provides a brief description of the designed liquid level control apparatus; Section 3 descries the experimental approach utilized to obtain the system mathematical model, Section 4 presents the design of the PID controller, experimental results and discussion are given in Section 5 and concluding remarks are provided in Section 6.

\section{System description}

The liquid level control system consisted of the following components: lower tank (reservoir), upper tank, $12 \mathrm{~V}$ centrifugal pump, microcontroller and the supporting electronics, an ultrasonic level sensor, and PC system with a custom-built MATLAB GUI. The upper and lower tanks were constructed of a $0.3 \mathrm{~cm}$ acrylic plates. The upper tank is $20 \mathrm{~cm}$ high and the lower tank is $30 \mathrm{~cm}$ high, see Figure 1 . The operating capacity of each tank is 8 liters of liquid. A combination of $1.9 \mathrm{~cm}$ diameter pipes and fittings served as an inlet and outlet for the liquid flow and allowed connection of the pump. A manual valve is used to vary the outlet flow rate, liquid flow from the upper tank 
to the lower tank. Overflow of the upper tank is prevented by an overflow hose that recirculated liquid into the reservoir tank below the upper tank. Figure 1 depicts the CAD design of the system and Figure 2 shows this fabricated liquid level control system. The block diagram depicted in Figure 3 summarizes the overall components of the automatic liquid level control system.
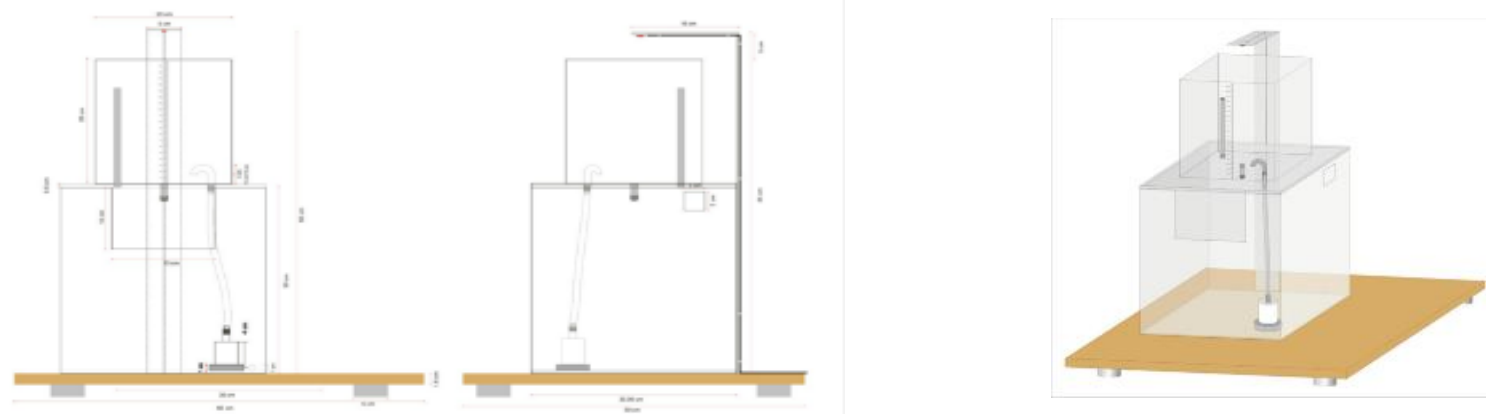

Fig. 1: CAD Design of the Liquid Level Control System.

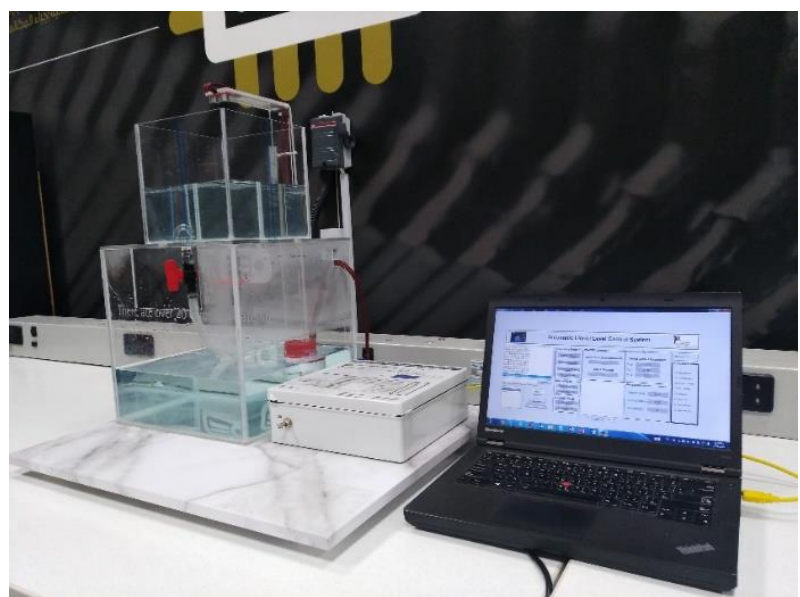

Fig. 2: Liquid Level Control System Apparatus.

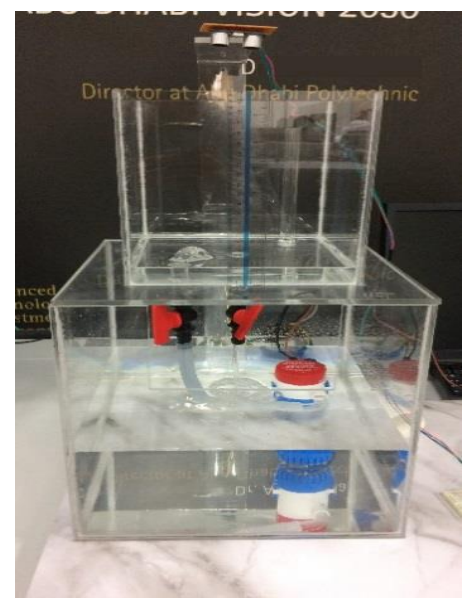

The MATLAB software was used for the design of the graphical user interface. The designed GUI searches for all the available serial ports and lists them on the menu at the bottom left of Figure 4. Then, the user can select the port corresponding to the microcontroller and establish the connection by pressing the connect button. The baud rate of the serial communication can also be adjusted. The GUI is designed to be as user friendly and self-explanatory as possible. It is divided into panels based on the available working modes. The supported working modes are, manual and automatic working modes. In the manual working mode, the operator can turn the pump On and Off. In the automatic mode, the operator can choose between ON/OFF controller and PID controller. The parameters of the selected controller can be adjusted using the available controls in the GUI as shown on Figure 4. The GUI communicates with the ATmega328 using serial communication protocol. It sends commands to the microcontrollers to select the working mode, set the control parameters, to run the pump, and to start plotting data. The microcontroller also sends data to be displayed on the GUI's indicators such as the current liquid level and the value of the control action.

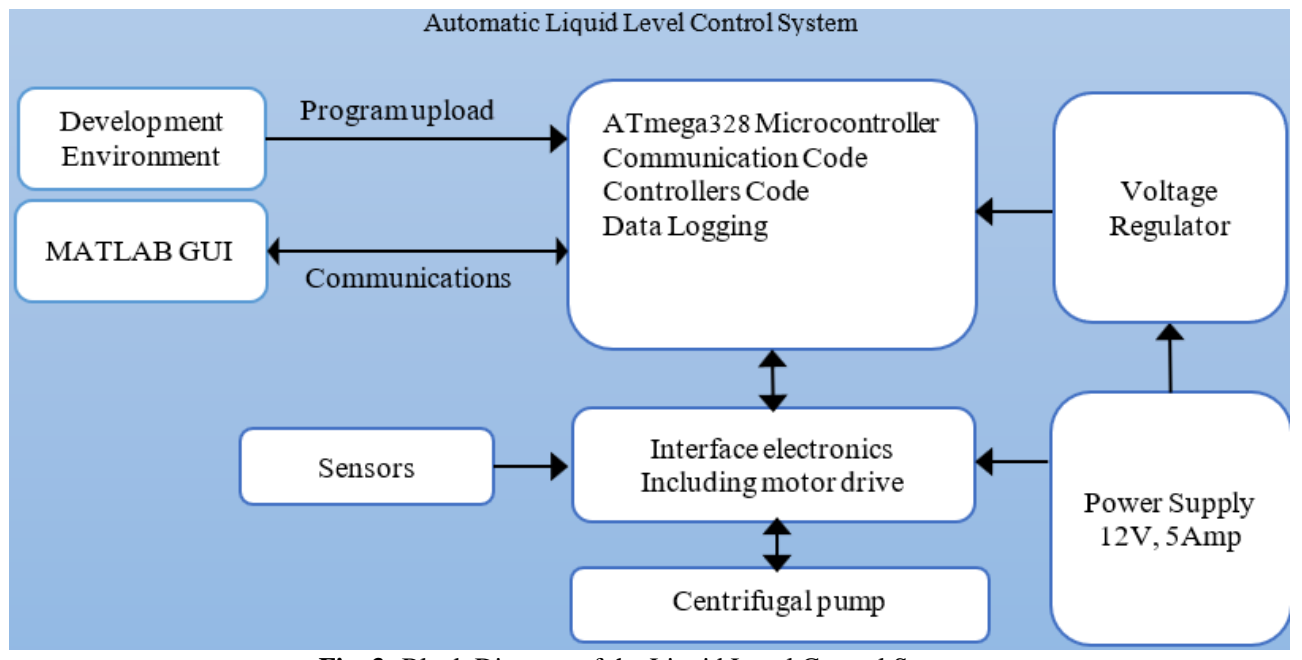

Fig. 3: Block Diagram of the Liquid Level Control System. 


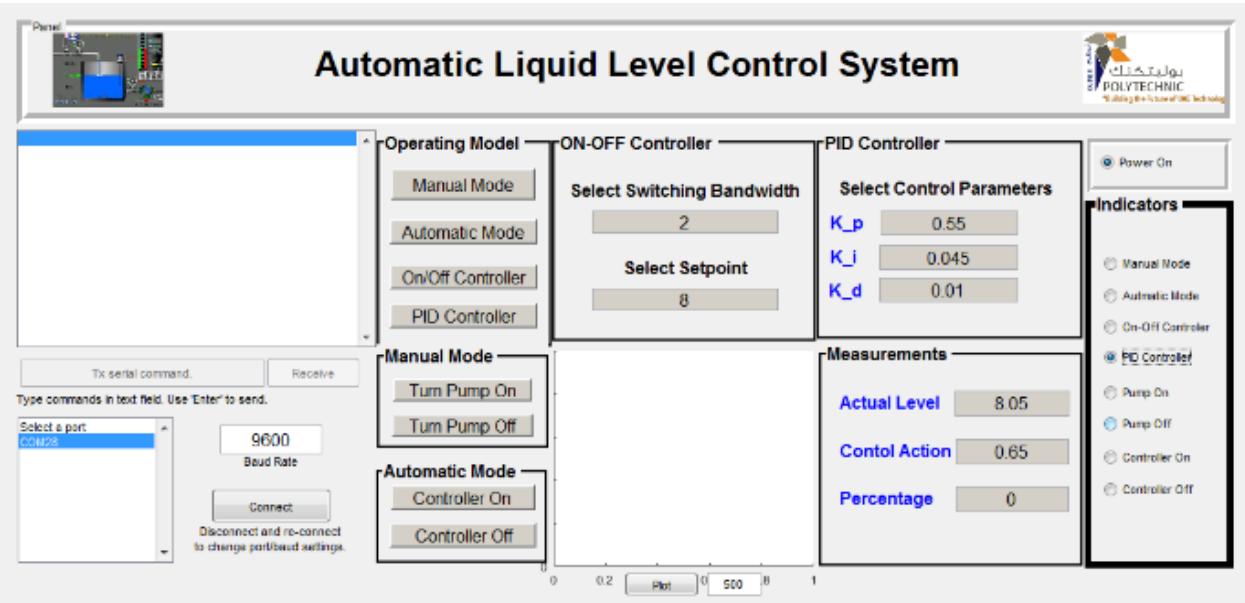

Fig. 4: GUI of the Liquid Level Control System.

\section{System mathematical model}

The plant mathematical model should describe the dynamics of the system, so that the controller designed based on the obtained model performs well. Due to the fact that system is a laboratory scale, there will be no significant change in the system's dynamics. Therefore, the system can be approximated by a single transfer function. The MATLAB Ident tool can accurately estimate the system transfer function form the open-loop experimental date. The centrifugal pump was operated on open-loop mode with 12 volts step input. The liquid level on the upper tank is sensed with the ultrasonic level sensor. The step input and the sensor output are logged in an excel file that is used with the Ident tool for obtaining the system transfer function. This process was repeated multiple times by varying the sampling time and the results are summarized in Table 1. The Ident tool is shown in Figure 5, after importing the time-domain data for the experiments. The figure also shows the estimated transfer functions.

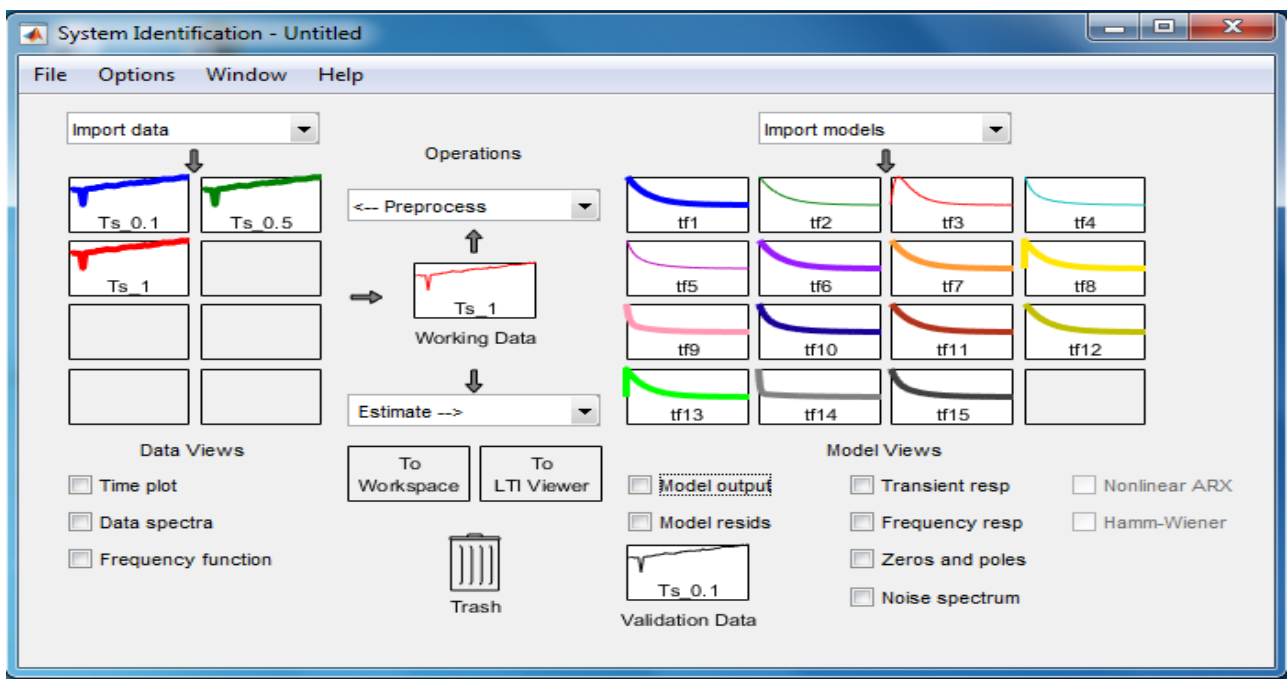

Fig. 5: System Transfer Functions Estimation with MATLAB System Identification Tool

The estimated transfer functions obtained from the experimental data is validated using the Validation Data of the Ident tool, the validation process starts by dragging the signal that was selected to estimate the transfer function to the box "Validation Data" on the main screen of the Ident tool and then proceeding to select "Model Output", whereby a window as shown in Figure 6 will open, it should be stressed that the signal used to validate must be the same as the signal used for the process estimation. Figure 7 shows the transfer function estimation report for the best fit and Figure 8 shows the output of the transfer function with the highest confidence level along with the experimental data.

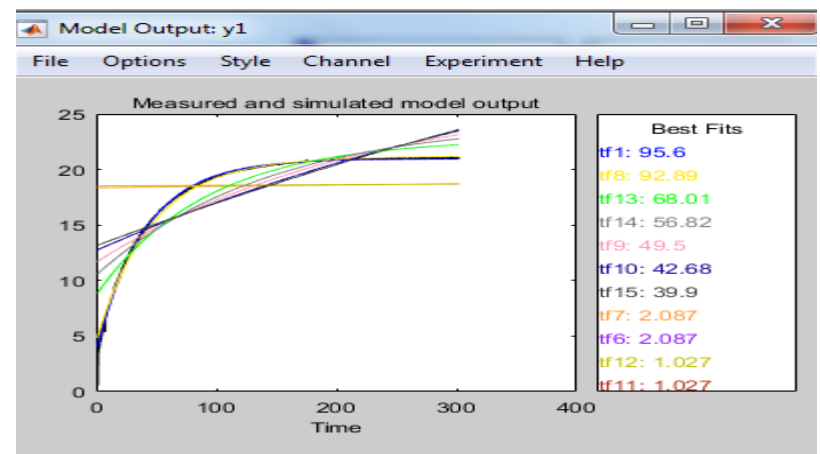

Fig. 6: Validation of the Obtained Transfer Functions. 


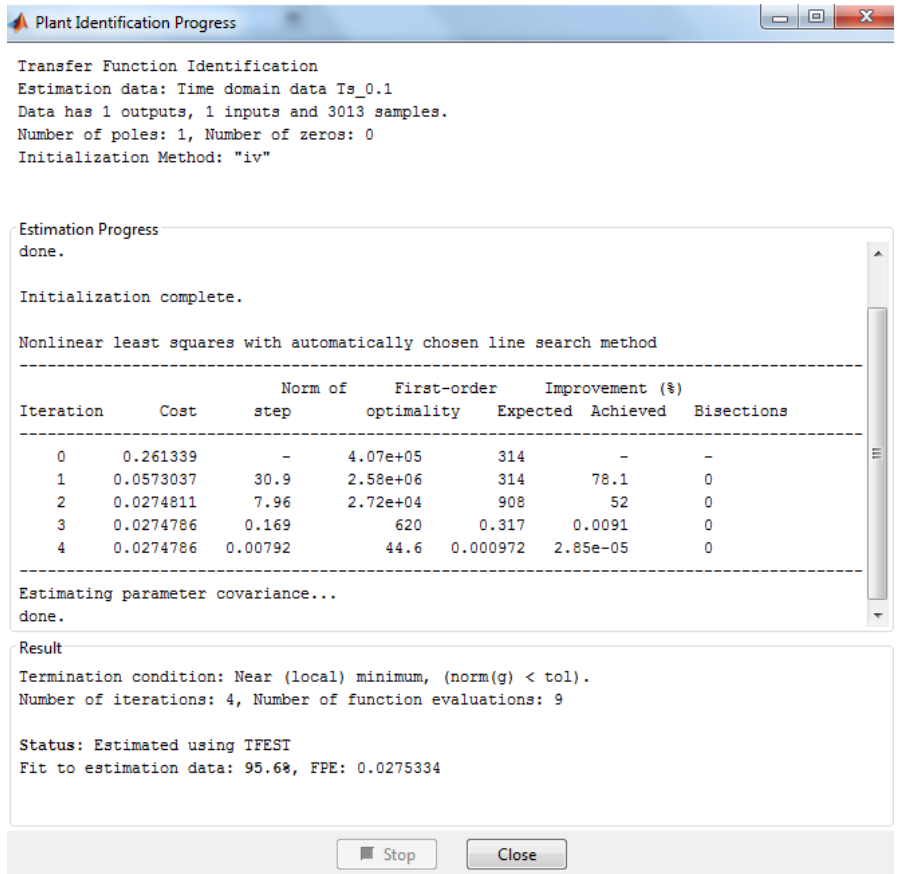

Fig. 7: Transfer Function Estimation Report.

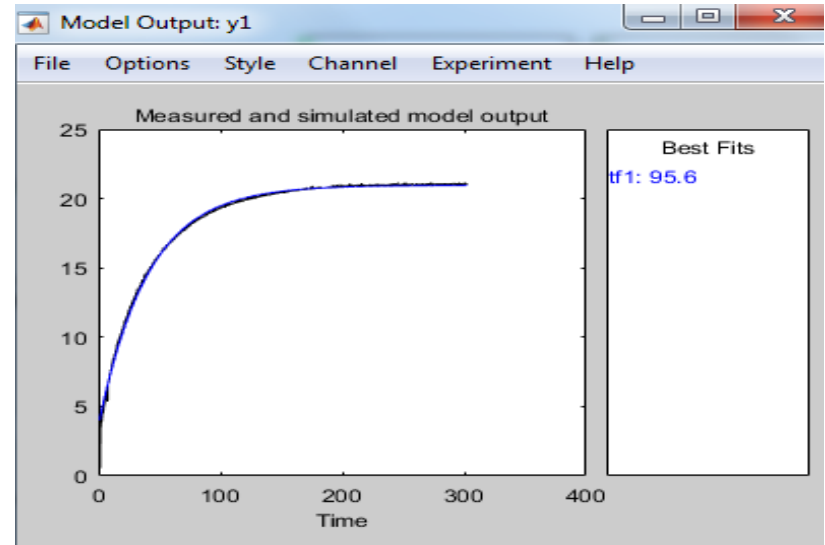

Fig. 8: Real System Output vs. The Output of the System Model with the Best Fit

After completing the transfer function estimation process with the aid of the system identification tool. The transfer function with the highest accuracy is exported to MATLAB Workspace, for which one must drag the desired transfer function to the box "To Workspace" located at the bottom of the main screen.

Table 1: Confidence Values Obtained with MATLAB Ident Tool

\begin{tabular}{llllll}
\hline $\begin{array}{l}\text { Step input parameters } \\
\text { Amplitude (v) }\end{array}$ & Sampling Time (s) & Percentage & & \\
& 1 Pole & 1 pol, 1 zero & 2 poles & 2 poles, 1 zero & 2 poles, 2 zeros \\
\hline $0-12$ & 0.01 & $56.82 \%$ & $39.9 \%$ & $-9.61 \%$ & $1.027 \%$ \\
$0-12$ & 0.05 & $95.6 \%$ & $68.01 \%$ & $91.2 \%$ & $48.5 \%$ \\
$0-12$ & 0.1 & $92.89 \%$ & $49.5 \%$ & $42.68 \%$ & $-1.456 \%$ \\
\hline
\end{tabular}

The maximum rate of $95.6 \%$ overlap is a transfer function of a single pole and no zeros. These results are in agreement with the experiment performed in level control of two tanks in [8]. The transfer function exported to MATLAB Workspace is given in Equation (1).

$$
G_{p}=\frac{0.06444}{s+0.0246}
$$

\section{Control design and implementation}

The implementation of the manual mode and the ON/OFF Controller is a straightforward process. Therefore, this section is devoted to the design of the PID controller. The MATLAB Control System Designer Toolbox presents an interactive way to automatically tune PID controllers based on classical methods. In addition, controllers can be tuned graphically by varying the root locus or the frequency responds. Figure 9 shows the main screen of the Control System Designer Toolbox. The system transfer function obtained in the previous section is imported to the Control System Designer Toolbox and the automatic PID tuning using robust response time is used to obtain the PID compensator transfer function as shown in Figure 10. The resulted transfer function is given in Equation (2). 


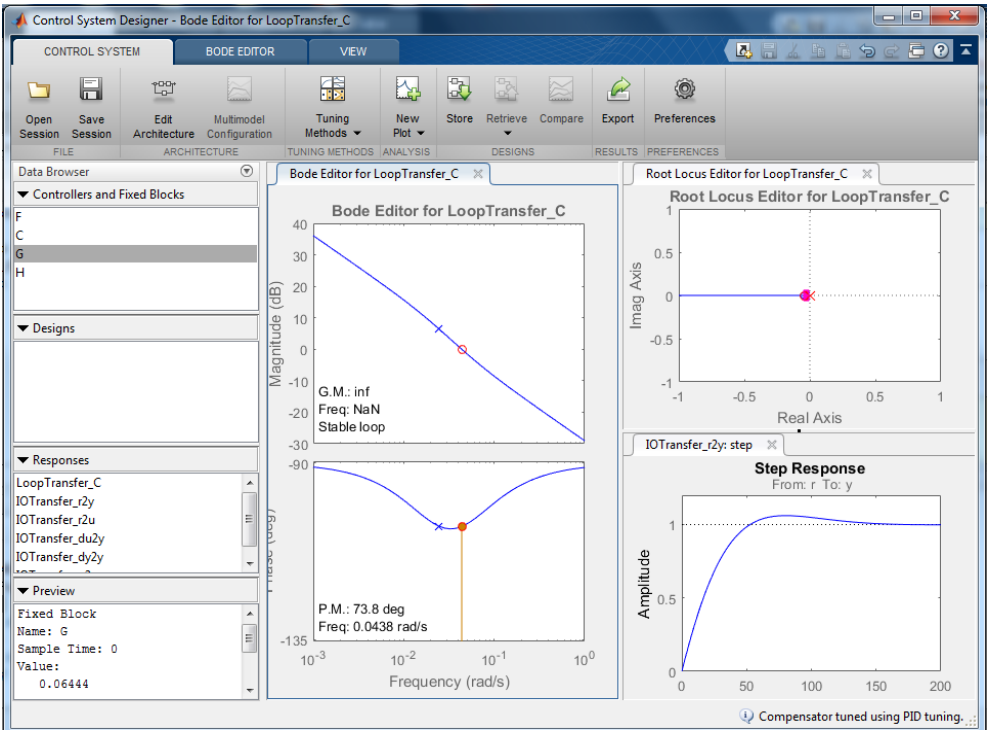

Fig. 9: Control System Designer Toolbox Main Window.

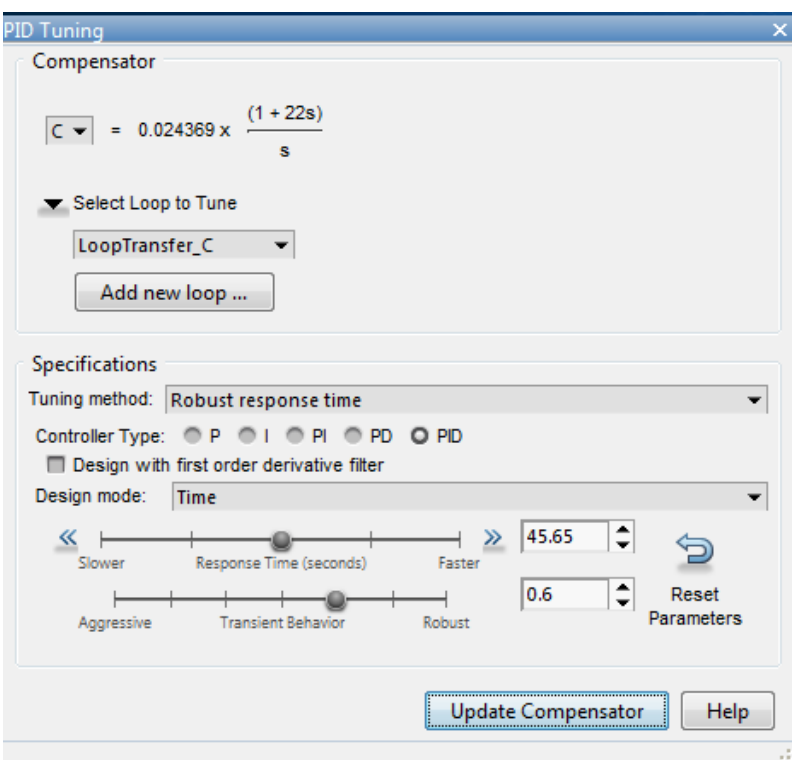

Fig. 10: Control System Designer PID Tuning Window.

$\mathrm{G}_{P I D}=\frac{0.54651(\mathrm{~s}+0.04459)}{\mathrm{s}}$

The values of the controller gains are $k_{p}=0.54651, k_{I}=0.04459$, and $k_{D}=0$. These values were subjected to fine-tuning when implemented on the system to achieve the desired response.

\section{Experiential results}

The PID controller that was designed in the previous section and a simple ON/OFF controller were implemented on the developed system's microcontroller. The experiential results for the ON/OFF controller and the PID controller are presented in this section.

The experimental result for the ON/OFF controller is shown in Figure 11. The setpoint is $10 \mathrm{~cm}$ and the differential gap is set to $2 \mathrm{~cm}$. The ON/OFF controller is operated as follows: the automatic control mode and ON/OFF controller are selected by activating the Automatic Mode switch and the On/Off controller switch from the Operating Mode panel of the GUI; then, the setpoint and the differential gap values can be selected; Finally, the controller is started by pressing the controller on switch.

The PID controller for the level control system is operated as follows: the automatic control mode and PID controller are selected by activating the Automatic Mode switch and the PID controller switch from the Operating Mode panel of the GUI; the PID controller parameters are selected and can be tuned online as desired; then, the value of the setpoint is selected. Finally, the controller is started by pressing the controller on switch. In the provided example, the setpoint was chosen to be $10,11,12,13$, and $18 \mathrm{~cm}$. The experimental result for the PID controller is depicted in Figure 12. The maximum overshoot is $10 \%$ and the settling time is $10 \mathrm{sec}$. 


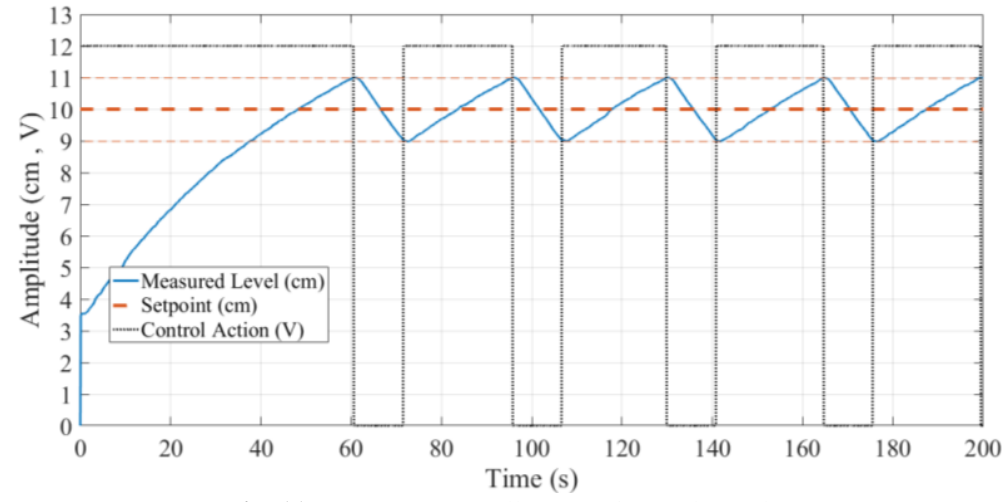

Fig. 11: ON/OFF Controller Experimental Results.

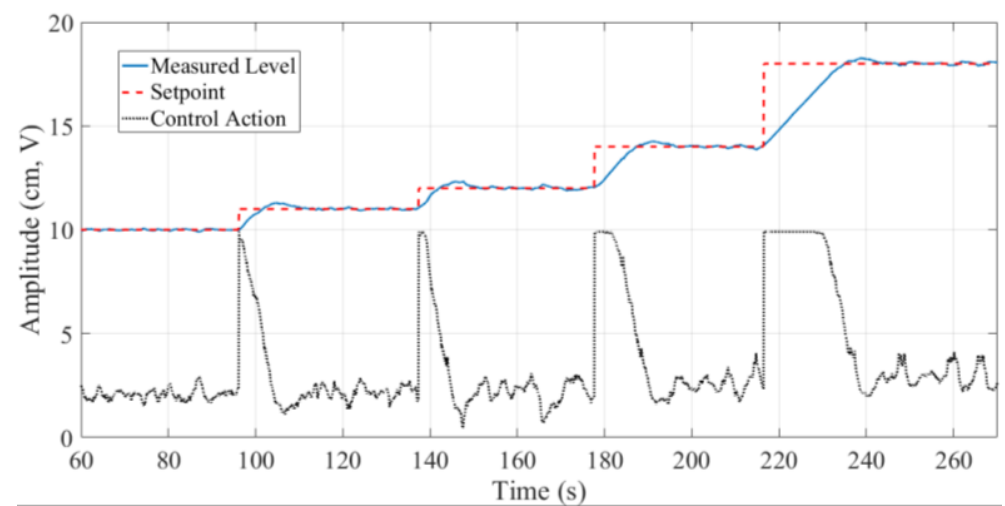

Fig. 12: PID Controller Experimental Results.

\section{Conclusion}

The work presented in this paper involved the design and development of an automatic liquid level control system. The system employs the use of advanced software tools for system identification and control design. Two types of controllers were implanted on the fabricated system; namely, an On/Off controller and a PID controller. A graphical user interface was specifically design and implemented for the interacting with the fabricated liquid level control system. Experimental results were presented to demonstrate the performance of the implemented On/Off and the PID controllers.

\section{References}

[1] J. D. Wagoner, N. F. Macia, "Automatic Liquid Level Controller Using A LabVIEW Based Pc", Department of Electronics \& Computer Technology Arizona State University East.

[2] T. Cheung, "Liquid-Level Control in Single Tanks and Cascades of Tanks with Proportional-Only and Proportional-Integral Feedback Controllers", Industrial \& Engineering Chemistry Fundamentals, 197918 (1), 15-21 https://doi.org/10.1021/i160069a004.

[3] M. Lee, J. Shin, "Constrained optimal control of liquid level loop using a conventional proportional-integral controller", Taylor and Francis, Chem. Eng. Commun. vol. 196, pp. 729-745, 2009. https://doi.org/10.1080/00986440802557393.

[4] L. Yu, W. Liu, and H. Wu, “Automatic Liquid Level Detection System Based on LabVIEW", Advanced Materials Research, 605-607, pp.1772-1775, 2012. https://doi.org/10.4028/www.scientific.net/AMR.605-607.1772.

[5] R. Paul, A. Sengupta, R. R. Pathak, "Wavelet based denoising technique for liquid level system", Measurement, vol. 46, pp. 1979-1994, 2013. https://doi.org/10.1016/j.measurement.2013.02.004.

[6] B. N. Getu and H. A. Attia, "Automatic water level sensor and controller system," 2016 5th International Conference on Electronic Devices, Systems and Applications (ICEDSA), Ras Al Khaimah, pp. 1-4, 2016. https://doi.org/10.1109/ICEDSA.2016.7818550.

[7] A. Piazzi and A. Visioli, "A Noncausal Approach for PID control", Journal of Process Control, 4 March 2006. https://doi.org/10.1016/j.jprocont.2006.03.001.

[8] M. A. Fellani and A. M. Gabaj, "PID controller design for two tanks liquid level control system using MATLAB", International Journal of Electrical and Computer Engineering (IJECE), vol. 5, ISSN 2088-8708, p. 336-342, 2015.

[9] S. Panda, B.K. Sahu, P.K. Mohanty, "Design and performance analysis of PID controller for an automatic voltage regulator system using simplified particle swarm optimization", Journal of the Franklin Institute, Volume 349, Issue 8, 2012, Pages 2609-2625, ISSN $0016-0032$. https://doi.org/10.1016/j.jfranklin.2012.06.008.

[10] S. Kumar, R. Negi, “A comparative study of PID tuning methods using anti-windup controller", Power Control and Embedded Systems (ICPCES) 2012 2nd International Conference on, pp. 1-4, 2012. https://doi.org/10.1109/ICPCES.2012.6508138.

[11] D. K. Sambariya, R. Prasad and D. Birla, "Design and performance analysis of PID based controller for SMIB power system using Firefly algorithm", 2015 2nd International Conference on Recent Advances in Engineering \& Computational Sciences (RAECS), Chandigarh, 2015, pp. 1-8.. https://doi.org/10.1109/RAECS.2015.7453394. 